\title{
New-onset nontuberculous mycobacterial pulmonary disease in bronchiectasis: tracking the clinical and radiographic changes
}

Nakwon Kwak', Jong Hyuk Lee², Hyung-Jun Kim¹, Sung A. Kim and Jae-Joon Yim ${ }^{1 *}$ (D)

\begin{abstract}
Background: The close association between bronchiectasis and nontuberculous mycobacterial pulmonary disease (NTM-PD) is well-known. However, the clinical impact of subsequent new-onset NTM-PD in bronchiectasis patients has not been elucidated. The aim of this study is to investigate the clinical courses and radiographic changes of patients with bronchiectasis in whom NTM-PD subsequently developed.

Methods: A total of 221 patients with bronchiectasis who had participated in a non-NTM bronchiectasis cohort between July 1st 2011 and August 31st 2019 at Seoul National University Hospital were included in this study. The data of patients in whom NTM-PD developed during this observation period were analyzed; specifically, changes in the Bronchiectasis Severity Index (BSI) and lesions on computerized tomography (CT) scan of the chest arising during the observation period.

Results: During the observation period, NTM was isolated from 35 patients. A total of 31 patients (14.0\%) satisfied the diagnostic criteria of NTM-PD. The median time from enrollment in the cohort to the development of subsequent NTM-PD was 37 months (Interquartile range [IQR], 18-78 months). Mycobacterium avium complex was the most common pathogen (80.6\%). Twelve patients underwent antibiotic treatment for NTM-PD with a median interval of 20 months (IQR, 13-30) from the time of NTM-PD diagnosis. When NTM-PD developed, the severity and extent of bronchiectasis, cellular bronchiolitis, and the extent of nodules worsened on CT scans, while BSI did not change.

Conclusions: NTM-PD can develop in previously negative bronchiectasis patients. It is associated with worsening radiographic lesions. Active screening of non-NTM bronchiectasis patients for new-onset NTM infection should be considered, especially if radiographic findings worsen. The BSI is not a reliable predictor of new-onset NTM-PD.
\end{abstract}

Trial registration: This study was performed at Seoul National University Hospital (NCT01616745).

Keywords: Mycobacterium, Incidence, Infection

\footnotetext{
* Correspondence: yimjj@snu.ac.kr

'Division of Pulmonary and Critical Care Medicine, Department of Internal Medicine, Seoul National University College of Medicine, 101 Daehak-Ro, Jongno-Gu, Seoul 110-744, South Korea

Full list of author information is available at the end of the article
}

(c) The Author(s). 2020 Open Access This article is licensed under a Creative Commons Attribution 4.0 International License, which permits use, sharing, adaptation, distribution and reproduction in any medium or format, as long as you give appropriate credit to the original author(s) and the source, provide a link to the Creative Commons licence, and indicate if changes were made. The images or other third party material in this article are included in the article's Creative Commons licence, unless indicated otherwise in a credit line to the material. If material is not included in the article's Creative Commons licence and your intended use is not permitted by statutory regulation or exceeds the permitted use, you will need to obtain permission directly from the copyright holder. To view a copy of this licence, visit http://creativecommons.org/licenses/by/4.0/. The Creative Commons Public Domain Dedication waiver (http://creativecommons.org/publicdomain/zero/1.0/) applies to the data made available in this article, unless otherwise stated in a credit line to the data. 


\section{Background}

Bronchiectasis is a chronic respiratory disease characterized by airway dilatation leading to impaired mucociliary clearance and persistent airway inflammation resulting in further airway damage [1]. It is a heterogeneous disease in terms of its etiology, co-existing morbidities, and prognosis $[1,2]$. Although bronchiectasis has been a neglected orphan disease, its incidence has been gradually increasing, especially in older age groups [3, 4]. Bronchiectasis is associated with increased mortality [4].

Nontuberculous mycobacterial pulmonary disease (NTMPD) can cause bronchiectasis directly. When it occurs in the presence of bronchiectasis, it can cause disease progression [1]. According to the U.S. Bronchiectasis Research Registry, about $60 \%$ of patients with bronchiectasis have had a previous history of NTM-PD or had NTM cultured from sputum [5]. NTM infection accounted for the etiology in $9.4 \%$ of patients with bronchiectasis in the U.S. population [6]. NTM-infected patients were diagnosed with bronchiectasis at a later age and had more dilated airways than those without NTM infection [5].

In patients with bronchiectasis from other causes, the anatomically altered bronchi are vulnerable to NTM infection $[1,7]$. The clinical impact of the development of NTM-PD in patients with underlying bronchiectasis has not been elucidated, especially in patients without cystic fibrosis [8]. In this study, we investigated the clinical course and radiographic changes in bronchiectasis patients with new-onset NTM-PD.

\section{Methods}

\section{Participants}

Our hospital has a prospective cohort of patients with bronchiectasis. All patients included in this analysis provided written informed consents to participate in the protocol, and the Institutional Review Board of Seoul National University Hospital approved the study (IRB No.1806-034-949). This study was conducted in accordance with the amended Declaration of Helsinki. A total of 221 patients with bronchiectasis who had participated in a non-NTM bronchiectasis cohort between July 1st 2011 and August 31st 2019 at Seoul National University Hospital were included (NCT01616745). Some of these patients had been included in previous studies [9-12]. The diagnosis of bronchiectasis was based on the radiographic features in thin-section computerized tomography (CT) scans as follows: 1) bronchial dilatation with respect to the accompanying pulmonary artery, 2) lack of tapering of the bronchial lumen, and 3) identification of bronchi within $1 \mathrm{~cm}$ of the pleural surface [13]. The absence of NTM infection was confirmed when negative growth of acid-fast bacilli (AFB) was proven at least two times more than 1 month apart after entry into the nonNTM bronchiectasis cohort.
Once diagnosed with bronchiectasis, the patients visited the clinics every 8-24 weeks according to their condition. AFB staining and mycobacterial culture of sputum were requested every year if patients could expectorate sputum and additional AFB staining and mycobacterial cultures could be requested at the discretion of the physician on duty based on symptomatic and radiographic changes. Pulmonary function tests and thin section CT of the chest were performed every 2 years.

When NTM was isolated from the sputum $\geq 2$ times or bronchoalveolar lavage and the clinical and radiographic findings were compatible with NTM-PD according the criteria suggested by American Thoracic Society (ATS)/ Infectious Diseases Society of America (IDSA) [14], a diagnosis of NTM-PD was made. These patients were included for the final analysis.

\section{Bronchiectasis severity index (BSI)}

The BSI score, which was developed to predict the risk of mortality, hospital admission, and exacerbation in patients with bronchiectasis [15], was measured at the time of initial enrollment of non-NTM bronchiectasis cohort as well as at the time of NTM-PD diagnosis. BSI score was calculated using the following variables: Age, body mass index (BMI), forced expiratory volume in $1 \mathrm{~s}$ (FEV1) \% predicted, hospital admission during the last 2 years, exacerbations during the last 1 year (a deterioration in three or more of cough, sputum volume/consistency, sputum purulence, breathlessness/exercise tolerance, fatigue/malaise and hemoptysis for at least $48 \mathrm{~h}$ ) [16], Medical Research Council (MRC) dyspnea score, colonization with Pseudomonas and/or other organisms, the number of involved lobes, or cystic bronchiectasis.

\section{CT scoring}

Chest CT scans were scored in terms of bronchiectasis (9 points maximum, with up to 3 points each assigned to degree of severity, extent, and mucus plugging), cellular bronchiolitis (6 points maximum, with up to 3 points each assigned to degree of severity and extent), cavity formation (9 points maximum, with up to 3 points each assigned to diameter, wall thickness, and extent). Nodules and consolidation were each scored with up to 3 points (absent, mild, moderate-to-severe) and the extent of involved segments (absent, 1-5 segments, 6-9 segments, or $>9$ segments), respectively [17]. All CT scans were reviewed by a board-certified radiologist (LJH).

\section{Statistical analysis}

Data are described as median values with interquartile ranges (IQRs) or proportions. For the categorical variables, the Chi-squared test and Fisher's exact test were used. The Wilcoxon signed-rank test was performed to compare continuous variables measured at the time of 
enrollment and at the time of NTM-PD diagnosis. All analyses were performed using commercial software (SPSS version $23.0^{\circ}$, SPSS Inc., Chicago IL, USA).

\section{Results}

The median follow-up duration for the 221 patients was 55 months (IQR 38-70). During this time, NTM was isolated from $35(15.8 \%)$ patients (30 patients from sputum and 5 patients from bronchoalveolar lavage. Among them, $31(14.0 \%)$ patients satisfied ATS/IDSA diagnostic criteria for NTM-PD. The patients with new-onset NTM-PD did not differ from the NTM-negative patients in terms of age, BMI, smoking history, previous history of tuberculosis, respiratory symptoms, initial FEV1\% predicted, bacterial colonization, radiographic findings and BSI. However, three or more exacerbations of bronchiectasis $(P=0.001)$ and hospital admission $(P=0.007)$ were more frequent in patients with new-onset NTMPD (Table 1). The median interval from enrollment into the cohort to NTM-PD development was 37 months (IQR 18-78 months) and the incidence rate for the development of NTM-PD was 32.3 per 1000 person-years
(95\% CI 22.7-45.9). The Kaplan-Meier plot of cumulative incidence of NTM-PD is illustrated in Fig. 1.

The most frequently encountered species was Mycobacterium avium (17, 54.8\%), followed by $M$. intracellulare (8, 25.8\%). M. abscessus subspecies abscessus was isolated in two patients. The other four patients were infected with two different species $(M$. avium/M. intracellulare in three patients and $M$. avium/M. abscessus subspecies massiliense in one patient). None of these patients were treated with a macrolide during the follow-up period, and the $M$. avium isolates from one patient showed macrolide resistance.

A total of $12(25.8 \%)$ of 31 patients received antibiotic treatment after a median of 47 months (IQR 16-103) from enrollment in the cohort and 20 months (IQR 13-30) from the diagnosis of NTM-PD because of radiographic aggravation (5 patients), symptomatic aggravation (5 patients) or both (2 patients) (Table 2). The mycobacterial species at the time of treatment was the same as that at the initial diagnosis in these patients and 7 patients achieved microbiological cure. BMI did not change from entry into the cohort (21.8 [IQR 19.4-23.8]) to the diagnosis of NTM-PD

Table 1 Baseline characteristics of patients of 31 patients with subsequent NTM-PD and 190 patient without subsequent NTM-PD

\begin{tabular}{|c|c|c|c|}
\hline & $\begin{array}{l}\text { Patients with subsequent } \\
\text { NTM-PD }(n=31)\end{array}$ & $\begin{array}{l}\text { Patients without subsequent } \\
\text { NTM-PD }(n=190)\end{array}$ & $P$ value \\
\hline Age, years, median [IQR] & $62(50-65)$ & $61(54-68)$ & 0.098 \\
\hline Female, n (\%) & $19(61.3)$ & $67(64.7)$ & 0.692 \\
\hline Body mass index, kg/m² median [IQR] & $21.4(19.4-23.7)$ & $22.0(19.8-24.2)$ & 0.419 \\
\hline Former or current smoker, $\mathrm{n}(\%)$ & $10(32.3)$ & $36(19.0)$ & 0.099 \\
\hline Previous history of tuberculosis, n (\%) & $12(38.7)$ & $68(35.8)$ & 0.841 \\
\hline Chronic obstructive pulmonary disease, n (\%) & $2(6.5)$ & $20(10.5)$ & 0.747 \\
\hline Asthma, n (\%) & $2(6.5)$ & $2(1.1)$ & 0.095 \\
\hline \multicolumn{4}{|l|}{ Respiratory symptom, n (\%) } \\
\hline Cough & $15(48.4)$ & $82(43.2)$ & 0.697 \\
\hline Sputum & $24(77.4)$ & $160(84.2)$ & 0.435 \\
\hline Hemoptysis & $11(35.5)$ & $42(22.1)$ & 0.116 \\
\hline Initial forced expiratory volume in $1 \mathrm{~s}(\%)$, median [IQR] & $96(72-103)$ & $88(72-104)$ & 0.519 \\
\hline Exacerbation during the last 1 year & & & 0.001 \\
\hline 0 & $22(71.0)$ & $128(67.4)$ & \\
\hline $1-2$ & $2(6.4)$ & $52(27.4)$ & \\
\hline 3 or more & $7(22.6)$ & $10(5.2)$ & \\
\hline Hospital admission during the last 2 years, $\mathrm{n}(\%)$ & $9(29.0)$ & $19(10.0)$ & 0.007 \\
\hline Pseudomonas colonization, n (\%) & 0 & $4(2.1)$ & $>0.999$ \\
\hline Colonization with other organisms, n (\%) & $2(6.5)$ & $17(9.0)$ & 0.646 \\
\hline$\geq 3$ lobes involved, n (\%) & $17(54.8)$ & $104(54.7)$ & $>0.999$ \\
\hline Presence of cavity, n (\%) & $2(6.5)$ & $19(10.0)$ & 0.746 \\
\hline Bronchiectasis severity score, median [IQR] & $4[3-5]$ & $4[2,7]$ & 0.963 \\
\hline
\end{tabular}

NTM-PD nontuberculous mycobacterial pulmonary disease, $I Q R$ inter-quartile range 


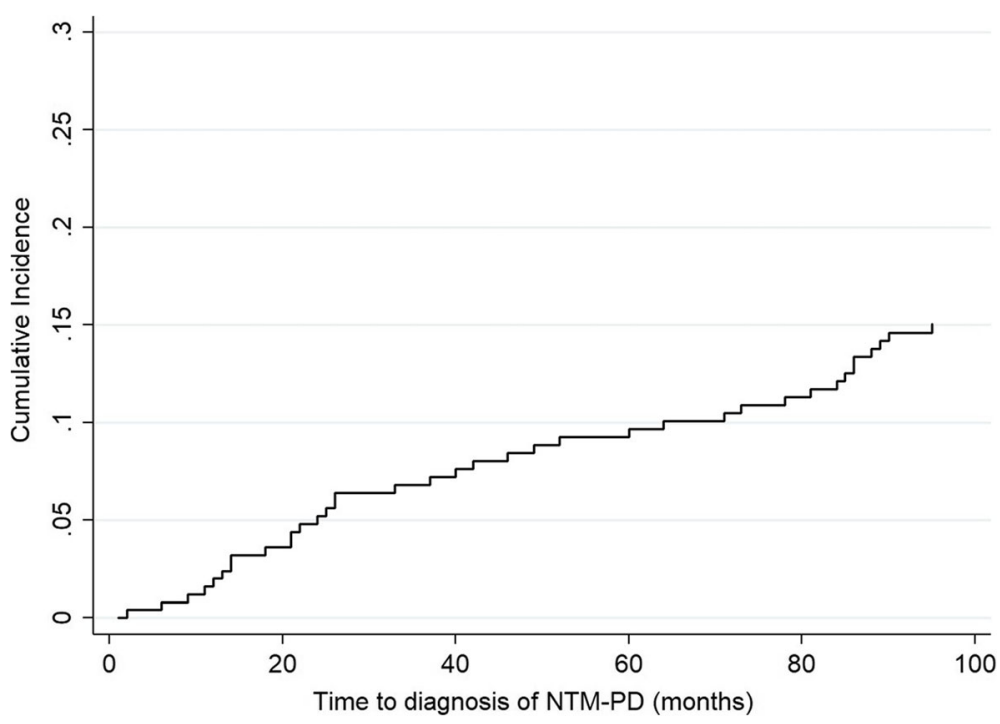

Fig. 1 Cumulative incidence curve of NTM-PD in non-NTM bronchiectasis

(21.8, [IQR 19.9-23.9]) $(P=.948)$. The median FEV1\% predicted was 93\% (IQR 70-102) and 96\% (IQR 76-107) at the time of non-NTM bronchiectasis cohort entry and NTM-PD diagnosis, respectively $(P=.06)$ (Table 2).

The BSI score at entry into the non-NTM bronchiectasis cohort was a median of 4 (IQR 2-7). It did not change at the time of NTM-PD diagnosis, with a median of 4 (IQR 3-6) (Table 3). The mean CT score increased from 7.6 (standard deviation $[\mathrm{SD}], 2.9$ ) on entry into the non-NTM bronchiectasis cohort to 9.1 (SD 2.4) at the time of NTM-PD diagnosis $(P=.002)$, while 12 of 31 patients in whom NTM-PD was subsequently diagnosed did not develop radiographic aggravation until diagnosis of NTM-PD. Specifically, severity $(P=.035)$ and mucus plugging $(P=.033)$ in bronchiectasis, severity $(P=.007)$

Table 2 Clinical features and outcomes of 31 patients after the diagnosis of NTM-PD

\begin{tabular}{|c|c|}
\hline $\begin{array}{l}\text { Time from the diagnosis of bronchiectasis to the } \\
\text { diagnosis of NTM-PD, months, median [IQR] }\end{array}$ & $37[18,78]$ \\
\hline \multicolumn{2}{|l|}{ NTM species, n (\%) } \\
\hline Mycobacterium avium complex & $25(80.6 \%)$ \\
\hline M. avium & $17(54.8)$ \\
\hline M. intracellulare & $8(25.8)$ \\
\hline \multicolumn{2}{|l|}{ Mycobacterium abscessus } \\
\hline M. abscessus & $2(6.5)$ \\
\hline \multicolumn{2}{|l|}{ Mixed infection } \\
\hline M. avium/ M. intracellulare & $3(9.7)$ \\
\hline M. avium/M. massiliense & $1(3.2)$ \\
\hline Treatment initiation, n (\%) & $12(25.8)$ \\
\hline Time to treatment initiation from the diagnosis of NTM-PD & $20(13-30)$ \\
\hline
\end{tabular}

and extent $(P=.039)$ of cellular bronchiolitis, and the extent of nodule distribution $(P=.034)$ increased significantly, while scores for cavity formation and consolidation were not changed (Table 4). The detailed scoring results of each patient are provided in Supplementary Table 1.

\section{Discussion}

For this study, we traced 221 patients participating in a non-NTM bronchiectasis cohort. During a median follow-up duration of 55 months, NTM-PD developed in 31 (14.0\%) after a median follow-up duration of 37 months. Diagnosis of NTM-PD was accompanied by worsening radiographic findings, while BSI did not change significantly.

Bronchiectasis and NTM-PD are closely linked. A recent meta-analysis reported the overall prevalence of NTM pulmonary infection in patients with bronchiectasis is $9.3 \%$ [18]. In this study, the prevalence of NTM-PD in patients with bronchiectasis was $14.0 \%$. The higher prevalence of NTM-PD in South Korea than in other areas, including the United States and Europe, might explain the relative abundance of NTM-PD in this cohort [19-21].

Though the correlation between bronchiectasis and NTM-PD is well-known, the causation is not fully established [22]. NTM infection causes bronchiectasis and it was regarded as an etiology of bronchiectasis in 9.4$18.0 \%$ of patients in two studies $[6,23]$. Conversely, the presence of bronchiectasis may predispose patients to acquire NTM, which may adhere to the damaged respiratory mucosal surface using fibronectin attachment protein [24]. Individuals with bronchiectasis are 50-75 times more likely to have NTM infection than those without bronchiectasis [3]. While the close linkage between these two entities has been recognized, the research 
Table 3 Changes in bronchiectasis severity index of 31 patients between the time of entry into the non-NTM bronchiectasis cohort and the time at the diagnosis of NTM-PD

\begin{tabular}{|c|c|c|c|}
\hline$n=31$ & $\begin{array}{l}\text { Entry of } \\
\text { non-NTM } \\
\text { BE cohort }\end{array}$ & $\begin{array}{l}\text { Diagnosis } \\
\text { of NTM-PD }\end{array}$ & $P$-value \\
\hline Age, years & & & 0.126 \\
\hline$<50$ & $7(22.6)$ & $2(6.5)$ & \\
\hline $50-69$ & $22(71.0)$ & $24(77.4)$ & \\
\hline 70-79 & $2(6.5)$ & $5(16.1)$ & \\
\hline$<80$ & 0 & 0 & \\
\hline Body mass index, $\mathrm{kg} / \mathrm{m}^{2}$ & & & 0.782 \\
\hline$<18.5$ & $4(12.9)$ & $4(12.9)$ & \\
\hline $18.5-25$ & $23(74.2)$ & $21(67.7)$ & \\
\hline $26-29$ & $4(12.9)$ & $6(19.4)$ & \\
\hline 30 or more & 0 & 0 & \\
\hline FEV1, \% predicted & & & 0.570 \\
\hline$>80$ & $21(67.7)$ & $24(77.4)$ & \\
\hline $50-80$ & $10(32.3)$ & $7(22.6)$ & \\
\hline $30-49$ & 0 & 0 & \\
\hline$<30$ & 0 & 0 & \\
\hline $\begin{array}{l}\text { Hospital admission during the last } \\
2 \text { years }\end{array}$ & & & 0.554 \\
\hline No & $22(71.0)$ & $25(80.9)$ & \\
\hline Yes & $9(29.0)$ & $6(19.4)$ & \\
\hline Exacerbation during the last 1 year & & & 0.317 \\
\hline 0 & $22(71.0)$ & $19(61.3)$ & \\
\hline $1-2$ & $2(6.4)$ & $6(19.4)$ & \\
\hline 3 or more & $7(22.6)$ & $6(19.4)$ & \\
\hline MRC dyspnea score & & & $>0.999$ \\
\hline $1-3$ & $31(100.0)$ & $31(100.0)$ & \\
\hline 4 & 0 & 0 & \\
\hline 5 & 0 & 0 & \\
\hline Pseudomonas colonization & & & $>0.999$ \\
\hline No & $31(100.0)$ & $31(100.0)$ & \\
\hline Yes & 0 & 0 & \\
\hline Colonization with other organisms & & & $>0.999$ \\
\hline No & $29(93.5)$ & $29(93.5)$ & \\
\hline Yes & $2(6.5)$ & $2(6.5)$ & \\
\hline $\begin{array}{l}3 \text { or more lobes involved } \\
\text { or cystic bronchiectasis }\end{array}$ & & & 0.434 \\
\hline No & $14(45.2)$ & $10(31.4)$ & \\
\hline Yes & $17(54.8)$ & $21(67.7)$ & \\
\hline Bronchiectasis severity score & & & 0.491 \\
\hline Mild & $21(67.7)$ & $17(54.8)$ & \\
\hline Moderate & $6(19.4)$ & $10(32.3)$ & \\
\hline Severe & $4(12.9)$ & $4(12.9)$ & \\
\hline Total score, median [IQR] & $4[2,7]$ & $4[3,6]$ & 0.903 \\
\hline
\end{tabular}

$B E$ bronchiectasis, NTM-PD nontuberculous mycobacterial pulmonary disease, IQR inter-quartile range
Table 4 Changes in CT score of 31 patients between the time of entry into the non-NTM bronchiectasis cohort and the time at the diagnosis of NTM-PD

\begin{tabular}{llll}
\hline Mean (SD) & $\begin{array}{l}\text { Entry of } \\
\text { non-NTM } \\
\text { BE cohort }\end{array}$ & $\begin{array}{l}\text { Diagnosis } \\
\text { of NTM-PD }\end{array}$ & $P$-value \\
\hline Bronchiectasis & $3.3(1.7)$ & $4.0(1.5)$ & 0.010 \\
$\quad$ Severity & $1.5(0.8)$ & $1.7(0.7)$ & 0.035 \\
Extent & $1.3(0.6)$ & $1.5(0.6)$ & 0.059 \\
$\quad$ Mucus plugging & $0.6(0.7)$ & $0.8(0.8)$ & 0.033 \\
Cellular bronchiolitis & $3.5(1.5)$ & $4.1(1.1)$ & 0.005 \\
$\quad$ Severity & $2.0(0.8)$ & $2.3(0.6)$ & 0.007 \\
$\quad$ Extent & $1.5(0.8)$ & $1.8(0.7)$ & 0.039 \\
Cavity & $0.3(1.3)$ & $0.3(1.3)$ & $>0.999$ \\
$\quad$ Diameter & $0.1(0.6)$ & $0.1(0.6)$ & $>0.999$ \\
$\quad$ Wall thickness & $0.1(0.5)$ & $0.1(0.5)$ & $>0.999$ \\
$\quad$ Extent & $0.1(0.3)$ & $0.1(0.3)$ & $>0.999$ \\
Nodules & $0.3(0.5)$ & $0.5(0.5)$ & 0.034 \\
Consolidation & $0.2(0.4)$ & $0.2(0.4)$ & $>0.999$ \\
Total score & $7.6(2.9)$ & $9.1(2.4)$ & 0.002 \\
\hline NTM-PD nontuberculous mycobacterial pulmonary disease, SD & \\
standard devation & & &
\end{tabular}

on the impact of NTM infection has mainly focused on bronchiectasis with cystic fibrosis $[25,26]$.

In our study, 31 out of 221 patients with non-NTM bronchiectasis were subsequently diagnosed with NTMPD over a median observation time of 37 months. Development of NTM-PD did not affect the BSI score. Clinical parameters included in the BSI score such as BMI, FEV1, number of hospital admissions, or the dyspnea score did not change. However, the radiographic severity worsened in terms of bronchiectasis, cellular bronchiolitis, and nodules. These results suggest that radiographic deterioration in patients with bronchiectasis could be a clue to the development of NTM-PD.

The current guidelines recommend mycobacterial cultures in patients with bronchiectasis only if initiation of long-term macrolide use is planned [27]. Given that subsequent development of NTM-PD (14.0\%) was not rare in non-NTM bronchiectasis, that it was accompanied by radiographic worsening, and that treatment for NTMPD was required in one-third of patients in our study, active screening for NTM infection among bronchiectasis patients is warranted [27]. In addition, considering that the symptoms and signs of bronchiectasis and NTM-PD overlap [8, 27], and that BSI score did not increase with development of subsequent NTM-PD in our study, periodic screening for NTM infection in bronchiectasis patients, regardless of planning macrolide therapy or symptomatic aggravation, should be performed. 


\section{Conclusions}

In conclusion, NTM-PD developing in previously NTMnegative bronchiectasis patients is accompanied by worsening radiographic findings. Active screening for NTM infection should be considered for all bronchiectasis patients, especially if radiographic lesions worsen.

\section{Supplementary Information}

The online version contains supplementary material available at https://doi. org/10.1186/s12890-020-01331-3.

Additional file 1: Supplementary Table. Detailed $C T$ scoring of 31 patients at the time of entry into the non-NTM bronchiectasis cohort and at the time of diagnosis of NTM-PD

\section{Abbreviations}

AFB: Acid-fast bacilli; ATS/IDSA: American Thoracic Society/Infectious Diseases Society of America; BMI: Body mass index; BSI: Bronchiectasis Severity Index; $\mathrm{CT}$ : Computerized tomography; FEV1: Forced expiratory volume in $1 \mathrm{~s}$; IQR: Interquartile range; MRC: Medical Research Council; NTMPD: Nontuberculous mycobacterial pulmonary disease

\section{Acknowledgments}

Not applicable

\section{Authors' contributions}

The authors meet criteria for authorship as recommended by the International Committee of Medical Journal Editors, were fully responsible for all content, and were involved at all stages of manuscript development Study conception and design: N. K and J.J.Y; Data collection: N. K, J.H.L, H.J.K, S.A.K and J.J.Y; Data Interpretation: N. K, J.H.L, H.J.K, S.A.K, and J.J.Y; and manuscript writing: N. K, J.H.L, H.J.K, S.A.K, and J.J.Y. As a corresponding author, J.J.Y has full access to all of the data in this study and takes complete responsibility for the integrity of the data and the accuracy of the data analysis. All authors have read and approved the manuscript.

\section{Funding}

No funding source.

\section{Availability of data and materials}

The dataset used are available from the corresponding author on reasonable request.

\section{Ethics approval and consent to participate}

This study was conducted in accordance with the amended Declaration of Helsinki and the Institutional Review Board of Seoul National University Hospital approved the protocol (IRB No.1806-034-949). All patients provided written informed consents to participate in the study.

\section{Consent for publication}

Not applicable.

\section{Competing interests}

The authors have no conflict of interest to declare.

\section{Author details}

${ }^{1}$ Division of Pulmonary and Critical Care Medicine, Department of Internal Medicine, Seoul National University College of Medicine, 101 Daehak-Ro, Jongno-Gu, Seoul 110-744, South Korea. ²Department of Radiology, Seoul National University College of Medicine, Seoul, South Korea.

Received: 6 April 2020 Accepted: 2 November 2020 Published online: 10 November 2020

\section{References}

1. Chalmers JD, Chang AB, Chotirmall SH, Dhar R, McShane PJ. Bronchiectasis. Nat Rev Dis Primers. 2018;4(1):45.
2. Chalmers JD, Aliberti S, Blasi F. Management of bronchiectasis in adults. Eur Respir J. 2015:45(5):1446-62.

3. Seitz AE, Olivier KN, Adjemian J, Holland SM, Prevots DR. Trends in bronchiectasis among medicare beneficiaries in the United States, 2000 to 2007. Chest. 2012;142(2):432-9.

4. Quint JK, Millett ER, Joshi M, Navaratnam V, Thomas SL, Hurst JR, Smeeth L, Brown JS. Changes in the incidence, prevalence and mortality of bronchiectasis in the UK from 2004 to 2013: a population-based cohort study. Eur Respir J. 2016;47(1):186-93.

5. Aksamit TR, O'Donnell AE, Barker A, Olivier KN, Winthrop KL, Daniels MLA, Johnson M, Eden E, Griffith D, Knowles M, et al. Adult patients with bronchiectasis: a first look at the US bronchiectasis research registry. Chest. 2017:151(5):982-92.

6. McShane PJ, Naureckas ET, Strek ME. Bronchiectasis in a diverse US population: effects of ethnicity on etiology and sputum culture. Chest. 2012 142(1):159-67

7. Bonaiti G, Pesci A, Marruchella A, Lapadula G, Gori A, Aliberti S. Nontuberculous mycobacteria in noncystic fibrosis bronchiectasis. Biomed Res Int. 2015;2015:197950.

8. Park IK, Olivier KN. Nontuberculous mycobacteria in cystic fibrosis and noncystic fibrosis bronchiectasis. Semin Respir Crit Care Med. 2015:36(2):217-24

9. Kim SJ, Yoon SH, Choi SM, Lee J, Lee CH, Han SK, Yim JJ. Characteristics associated with progression in patients with of nontuberculous mycobacterial lung disease : a prospective cohort study. BMC Pulm Med. 2017; 17(1):5.

10. Kwak N, Lee CH, Lee HJ, Kang YA, Lee JH, Han SK, Yim JJ. Non-tuberculous mycobacterial lung disease: diagnosis based on computed tomography of the chest. Eur Radiol. 2016;26(12):4449-56.

11. Lee AR, Lee J, Choi SM, Seong MW, Kim SA, Kim M, Chae KO, Lee JS, Yim JJ. Phenotypic, immunologic, and clinical characteristics of patients with nontuberculous mycobacterial lung disease in Korea. BMC Infect Dis. 2013;13:558.

12. Park J, Cho J, Lee CH, Han SK, Yim JJ. Progression and treatment outcomes of lung disease caused by Mycobacterium abscessus and Mycobacterium massiliense. Clin Infect Dis. 2017;64(3):301-8.

13. Hansell DM, Bankier AA, MacMahon H, McLoud TC, Muller NL, Remy J. Fleischner society: glossary of terms for thoracic imaging. Radiology. 2008; 246(3):697-722

14. Griffith DE, Aksamit T, Brown-Elliott BA, Catanzaro A, Daley C, Gordin F, Holland SM, Horsburgh R, Huitt G, lademarco MF, et al. An official ATS/IDSA statement: diagnosis, treatment, and prevention of nontuberculous mycobacterial diseases. Am J Respir Crit Care Med. 2007;175(4):367-416.

15. Chalmers JD, Goeminne P, Aliberti S, McDonnell MJ, Lonni S, Davidson J, Poppelwell L, Salih W, Pesci A, Dupont LJ, et al. The bronchiectasis severity index. An international derivation and validation study. Am J Respir Crit Care Med. 2014;189(5):576-85.

16. Hill AT, Haworth CS, Aliberti S, Barker A, Blasi F, Boersma W, Chalmers JD, De Soyza A, Dimakou K, Elborn JS, et al. Pulmonary exacerbation in adults with bronchiectasis: a consensus definition for clinical research. Eur Respir J. 2017;49(6). https://erj.ersjournals.com/content/49/6/1700051.

17. Kim HS, Lee KS, Koh WJ, Jeon K, Lee EJ, Kang H, Ahn J. Serial CT findings of Mycobacterium massiliense pulmonary disease compared with Mycobacterium abscessus disease after treatment with antibiotic therapy. Radiology. 2012;263(1):260-70.

18. Chu H, Zhao L, Xiao H, Zhang Z, Zhang J, Gui T, Gong S, Xu L, Sun X. Prevalence of nontuberculous mycobacteria in patients with bronchiectasis: a meta-analysis. Arch Med Sci. 2014;10(4):661-8.

19. Henkle E, Hedberg K, Schafer S, Novosad S, Winthrop KL. Population-based incidence of pulmonary Nontuberculous mycobacterial disease in Oregon 2007 to 2012. Ann Am Thorac Soc. 2015:12(5):642-7.

20. Lee H, Myung W, Koh WJ, Moon SM, Jhun BW. Epidemiology of Nontuberculous mycobacterial infection, South Korea, 2007-2016. Emerg Infect Dis. 2019;25(3):569-72.

21. Wagner D, Lipman M, Cooray S, Ringshausen FC, Morimoto K, Koh WJ, Thomson R. Global Epidemiology of NTM Disease (Except Northern America). In: Nontuberculous Mycobacterial Disease: Springer; 2019. p. 163-260. https://link.springer.com/book/10.1007/978-3-319-93473-0.

22. Griffith DE, Aksamit TR. Bronchiectasis and nontuberculous mycobacterial disease. Clin Chest Med. 2012;33(2):283-95.

23. Kadowaki T, Yano S, Wakabayashi K, Kobayashi K, Ishikawa S, Kimura M, Ikeda T. An analysis of etiology, causal pathogens, imaging patterns, and 
treatment of Japanese patients with bronchiectasis. Respir Investig. 2015; 53(1):37-44.

24. Middleton AM, Chadwick MV, Nicholson AG, Dewar A, Groger RK, Brown EJ, Wilson R. The role of Mycobacterium avium complex fibronectin

attachment protein in adherence to the human respiratory mucosa. Mol Microbiol. 2000;38(2):381-91.

25. Olivier KN, Weber DJ, Wallace RJ Jr, Faiz AR, Lee JH, Zhang Y, Brown-Elliot BA, Handler A, Wilson RW, Schechter MS, et al. Nontuberculous mycobacteria. I: multicenter prevalence study in cystic fibrosis. Am J Respir Crit Care Med. 2003;167(6):828-34.

26. Roux AL, Catherinot E, Ripoll F, Soismier N, Macheras E, Ravilly S, Bellis G, Vibet MA, Le Roux $E$, Lemonnier $L$, et al. Multicenter study of prevalence of nontuberculous mycobacteria in patients with cystic fibrosis in France. J Clin Microbiol. 2009;47(12):4124-8.

27. Polverino E, Goeminne $\mathrm{PC}$, McDonnell MJ, Aliberti S, Marshall SE, Loebinger MR, Murris M, Canton R, Torres A, Dimakou K, et al. European Respiratory Society guidelines for the management of adult bronchiectasis. Eur Respir $J$. 2017;50(3). https://erj.ersjournals.com/content/50/3/1700629.

\section{Publisher's Note}

Springer Nature remains neutral with regard to jurisdictional claims in published maps and institutional affiliations.

Ready to submit your research? Choose BMC and benefit from:

- fast, convenient online submission

- thorough peer review by experienced researchers in your field

- rapid publication on acceptance

- support for research data, including large and complex data types

- gold Open Access which fosters wider collaboration and increased citations

- maximum visibility for your research: over $100 \mathrm{M}$ website views per year

At $\mathrm{BMC}$, research is always in progress.

Learn more biomedcentral.com/submissions 